\title{
Geschlechtsspezifische Unterschiede der freien Aminosäuren im Urin von Erwachsenen
}

\author{
Von N. Liappis \\ Aus der Universitäts-Kinderklinik (Direktor: Prof. Dr. H. Hungerland) Bonn
}

(Eingegangen am 20. Dezember 1972/26. Februar 1973)

Bei 102 gesunden Erwachsenen wurden die freien Aminosäuren im 24-h-Urin quantitativ bestimmt. Der Urin wurde mit $50 \mathrm{~g} / \mathrm{l}$ Sulfosalicylsäure enteiweißt; die Auftrennung der Aminosäuren erfolgte durch Chromatographic an Ionenaustauschersäulen. Es wurden 33 Männer (Alter 21 bis 43 Jahre) und 69 Frauen (Alter 18 bis 46 Jahre, davon 39 ohne Einnahme und 30 mit Einnahme von oralen Kontrazeptiva) untersucht. Es wurden folgende Befunde erboben:

1. Frauen, die keine oralen Kontrazeptiva einnahmen, schieden in $24 \mathrm{~h}$ signifikant weniger Arginin, Histidin, o-Phosphoäthanolamin und Valin aus als Männer.

2. Frauen, die keine oralen Kontrazeptiva einnahmen, schieden in $24 \mathrm{~h}$ signifikant mehr Glycin aus als Männer.

3. Frauen, die orale Kontrazeptiva einnahmen, schieden in $24 \mathrm{~h}$ signifikant weniger Arginin, Ornithir, 0 -Phosphoäthanolamin, Tyrosin und Valin aus als Männer.

4. Frauen, die orale Kontrazeptiva einnahmen, schieden signifikant weniger Glycin aus als Frauen, die orale Kontrazeptiva nicht einnahmen.

5. Wurden die in $24 \mathrm{~h}$ ausgeschiedenen Aminosäurenmengen auf das Körpergewicht bezogen, so zeigte sich, daß nur in der GlycinAusscheidung ein Unterschied in so weit bestand, als Frauen, die keine oralen Kontrazeptiva einnahmen, signifikant mehr Glycin ausschieden als alle anderen Männer und Frauen.

\section{Sex-specific differences in the free amino acids in adult urine}

Free amino acids were determined quantitatively in the $24 \mathrm{~h}$ urine of 33 healthy men (ages 21-43) and 69 healthy women (ages 18-46). 30 of the women were taking oral contraceptives. Urine was deproteinised by adding an equal volume of $50 \mathrm{~g} / \mathrm{l}$ sulphosalicylic acid, and the amino acids were separated by ion exchange column chromatography. Results:

1. Compared with the male group, the 39 females not taking oral contraceptives had statistically significant lower values for the excretion of the following amino acids: arginine, histidine, phosphoethanolamine and valine.

2. Compared with the males, the females not taking oral contraceptives showed a statistically significant higher value only for the excretion of glycine.

3. Compared with the males, the females taking oral contraceptives had statistically significant lower values for the excretion of the following amino acids: arginine, ornithine, phosphoethanolamine, tyrosine and valine.

4. Compared with the females not taking oral contraceptives, the females taking oral contraceptives showed a statistically significant lower excretion only of glycine.

5. The calculation of the excretion of the free amino acids in the $24 \mathrm{~h}$ urine per kilogram of the body weight revealed that only glycine showed a statistically significant higher excretion in the urine of the females not taking oral contraceptives as compared to the male group and the female group taking oral contraceptives. All the other examined amino acids showed a nearly constant pattern without sex-specific differences.

Veränderungen der Aminosäurenausscheidung im Urin treten vor allem bei Lebererkrankungen, Nephropathien und bei kongenitalen Stoffwechselstörungen auf. Die gleichzeitige Bestimmung der Konzentration einer Mehrzahl freier Aminosäuren im Blutserum bzw. im Urin ist erst mit Hilfe der modernen Techniken der Aminosäurenanalyse ermöglicht worden. So konnten in den letzten $1.0 \mathrm{Jahren}$ mehr als 40 angeborene Störungen des Aminosäurestoffwechsels entdeckt werden. Sie äußern sich in spezifischen Hyperaminoaciduriebzw. Hyperaminoacidämie-Mustern, die z. T. Schwachsinn zur Folge haben können bzw. zum Tode führen. In zahlreichen Arbeiten wird über das Verhalten der Aminosäuren im Blut und Urin des gesunden und kranken Menschen berichtet. Zusammenfassungen über die Bedeutung der Aminoacidurie und Hyperamino- acidurie finden sich bei BICKEL und Souchon (1), Bigivood et al. (2), Chisolm (3), Harris (4), Harrison und Harrison (5), JonXis (6), LinNeweh (7), Schreier (8), SNYDERMaN (9), SNYDERMaN und Holt (10), Soupart (11) und anderen.

Eine umfassende Übersicht über die verschiedenen Analysenmethoden wurde von NIEDERwIESER und CuRTIUs (12) zusammengestellt. Im Vergleich zu den quantitativen mikrobiologischen, kolorimetrischen und enzymatischen Analysenmethoden für einzelne Aminosäuren in biologischen Flüssigkeiten besitzt die Chromatographie an Ionenaustauschersäulen $(13-16)$ bei weitem die größte Bedeutung. Sie ermöglicht die gleichzeitige Bestimmung einer erheblichen Anzahl von Aminosäuren. So finden sich im Urin bei der automatischen, chromatographischen Trennung an Ionen- 
austauschersäulen nach STEIN und Moore (17) bis zu 80 mit Ninhydrin reagierende Verbindungen, von denen 29 Aminosäuren darstellen (18).

Während die Normalkonzentration der freien.Aminosäuren im Blutserum von Erwachsenen schon eingehend untersucht wurde (19-22), ist über die Ausscheidung der freien Aminosäuren im 24-h-Urin nur eine geringe Anzahl von Untersuchungen durchgeführt worden (23-25).

In der vorliegenden Arbeit wurde zur Aufstellung von Normalwerten für die Ausscheidung der freien Aminosäuren im 24-h-Urin von Erwachsenen das säulenchromatographische Verfahren mit dem AminosäurenAuto-Analysator der Fa. Technicon (Frankfurt/Main) angewandt. Zusätzlich wurde derEinfluß der oralen Kontrazeptiva auf die Aminosäurenausscheidung im 24-h-Urin untersucht.

\section{Methodik}

Die Bestimmung der freien Aminosäuren im 24-h-Urin wurde bei insgesamt 33 Männern und 69 Frauen, bei denen für Leberschäden und Stoffwechselstörungen keine Anhaltspunkte bestanden, durchgeführt. Die weiblichen Normalpersonen wurden in die Gruppe ohne Einnahme von oralen Kontrazeptiva ( $n=39$ ) und in die Gruppe mit Einnahme von oralen Kontrazeptiva $(n=30)$ aufgeteilt. Der Urin wurde nach BrEUER et al. (19) mit $50 \mathrm{~g} / 1$ Sulfosalicylsäure 1:1 $(\nabla / v)$ enteiweißt. Die Aufbewahrung erfolgte bei $-20^{\circ} \mathrm{C}$.

Die säulenchromatographische Bestimmung der Aminosäuren (26) wurde mit dem Aminosäuren-Auto-Analysator der Fa. Technicon durchgeführt. Nach dem Auftragen der enteiweißten Urinproben mit Norleucin als Standard-Aminosäure, wurde unter Verwendung der Autograd-Technik $(27,28)$ mit verschiedenen Puffersystemen eluiert.

Während der Analysendauer von etwa $22 \mathrm{~h}$ betrug die Arbeitstemperatur der Chromatographiesäulen $60^{\circ} \mathrm{C}$, die Durchflußmenge $0,5 \mathrm{ml} / \mathrm{min}$ und der Druck 300 bis 400 pounds/square inch. Der Nachweis der freien Aminosäuren erfolgte durch Reaktion mit Ninhydrin-Hydrindantin-Reagenz.

Die Auswertung der Aminosäurenchromatogramme wurde halbautomatisch mit einem Technicon Integrator/Kalkulator durch Vergleich mit Chromatogrammen einer Standard-AminosäurenLösung, die 23 Aminosäuren ( $F$. Cyclo-Chemical-Corporation, Los Angeles, California und Fa. Serva, Heidelberg) in Konzentrationen von je $2,5 \mathrm{mmol} / \mathrm{l}$ enthielt, durchgeführt. Mit Hilfe dieser Testchromatogramme wurden auch die einzelnen Gipfel der Chromatogramme im Urin den entsprechenden Aminosäuren zugeordnet.

Die Kontrolle der Methode erfolgte durch die Bestimmung von 10 Analysen im Urin eines Erwachsenen. Die Standardabweichung $( \pm s)$ und der Variationskoeffizient für die einzelnen, freien Aminosäuren im Urin betrug wie folgt:

Äthanolamin $624,7 \pm 22,6 \mu \mathrm{mol} / 1$ (VK 3,62\%)

Alanin 577,7 $\pm 9,2 \mu \mathrm{mol} / 1$ (VK 1,59\%)

$\beta$-Aminoisobuttersäure $137,5 \pm 4,7 \mu \mathrm{mol} / \mathrm{l}(\mathrm{VK} 3,42 \%)$

Arginin $23,4 \pm 2,2 \mu \mathrm{mol} / 1$ (VK 9,40\%)

Carnosin $51,8 \pm 4,3 \mu \mathrm{mol} / 1$ (VK 8,30\%)

Citrullin $36,8 \pm 2,5 \mu \mathrm{mol} / 1$ (VK 6,79\%)

Cystin $150,9 \pm 7,1 \mu \mathrm{mol} / 1$ (VK 4,71\%)

Glycin 5600,5 $\pm 32,7 \mu \mathrm{mol} / 1$ (VK $0,58 \%)$

Histidin $2880,2 \pm 19,2 \mu \mathrm{mol} / 1$ (VK $0,67 \%$ )

Isoleucin $25,6 \pm 1,9 \mu \mathrm{mol} / 1$ (VK 7,42\%)

Leucin $67,0 \pm 5,4 \mu \mathrm{mol} / 1$ (VK 8,06\%)

Lysin $1791,0 \pm 23,1 \mu \mathrm{mol} / 1$ (VK 1,29\%)

Methionin 14,7 $\pm 1,35 \mu \mathrm{mol} / 1$ (VK 9,18\%)

Ornithin 52,5 $\pm 4,0 \mu \mathrm{mol} / \mathrm{l}(\mathrm{VK} 7,62 \%)$

Phenylalanin 84,8 $\pm 3,2 \mu \mathrm{mol} / 1$ (VK 3,77\%)
o-Phosphoäthanolamin $20,1 \pm 1,3 \mu \mathrm{mol} / 1$ (VK 6,47\%)

Serin $690,2 \pm 17,0 \mu \mathrm{mol} / 1$ (VK 2,46\%)

Threonin $1077,2 \pm 33,6 \mu \mathrm{mol} / 1$ (VK 3,12\%)

Tyrosin $447,6 \pm 15,7 \mu \mathrm{mol} / 1$ (VK 3,51\%)

Valin $52,4 \pm 3,2 \mu \mathrm{mol} / 1$ (VK $6,11 \%$ ).

\section{Ergebnisse}

In Tabelle 1 ist der Mittelwert, die Standardabweichung und der Schwankungsbereich von 20 freien Aminosäuren im 24-h-Urin von 102 erwachsenen Normalpersonen geschlechtsspezifisch dargestellt. In Tabelle 2 wurden die Werte der einzelnen, freien Aminosäuren im 24-h-Urin der untersuchten, gesunden Erwachsenen pro Kilogramm Körpergewicht angegeben und wiederum der Mittelwert, die Standardabweichung und der Schwankungsbereich dargestellt.

Bei den weiblichen Normalpersonen wurde die Einnahme von Hormonen (orale Kontrazeptiva) berücksichtigt.

Die Auswertung der Ergebnisse erfolgte nach dem tTest für nicht-gepaarte Daten von SruDENT. Unterschiede wurden als statistisch signifikant angesehen, wenn der p-Wert kleiner als 0,02 war (s. Tab. 1 und 2).

In Tabelle 1 und 2 sind zusätzlich die in der Literatur beschriebenen Ergebnisse $(23-25,29)$ angegeben.

Aus unseren Untersuchungen ergaben sich folgende Befunde:

1. Mit Ausnahme von Glycin und Carnosin war die Ausscheidung der übrigen 18 untersuchten freien Aminosäuren im 24-h-Urin der weiblichen Normalpersonen ohne Einnahme oraler Kontrazeptiva niedriger als bei den männlichen Normalpersonen.

2. Signifikant niedriger war die Ausscheidung der freien Aminosäuren im 24-h-Utin dieser weiblichen Normalpersonen bei folgenden Aminosäuren: Arginin, Histidin, o-Phosphoäthanolamin und Valin; signifikant erhöht zeigte sich nur Glycin.

3. Bedeutend niedriger als bei den männlichen Normalpersonen war die Ausscheidung der freien Aminosäuren im 24-h-Urin dieserr weiblichen Normalpersonen bei folgenden Aminosäuren: Äthanolamin, $\beta$-Aminoisobuttersäure, Cystin, Leucin, Ornithin, Serin und Tyrosin.

4. Im Vergleich $z u$ den männlichen Normalpersonen zeigte sich mit Ausnahme der $\dot{\beta}$-Aminoisobuttersäure die Ausscheidung aller anderen untersuchten freien Aminosäuren im 24-h-Urin der weiblichen Normalpersonen mit Einnahme von oralen Kontrazeptiva niedriger.

5. Signifikant niedriger war die Ausscheidung folgender freier Aminosäuren im 24-h-Utin dieser weiblichen Normalpersonen mit Einnahme von oralen Kontrazeptiva: Arginin, Ornithin, o-Phosphoäthanolamin, Tyrosin und Valin.

6. Bedeutend niedriger als bei den männlichen Normalpersonen zeigte sich die Ausscheidung von Äthanölamin, Alanin, Citrullin, Cystin, Glycin, Histidin, Isoleucin, Leucin, Lysin, Phenylalanin und Serin bei diesen weiblichen Normalpersonen. 
Tab. 1

Ausscheidung der freien Aminosäuren im 24-h-Urin gesunder Mănner $(n=33)$ und Frauen $(n=69)$ - aufgeteilt in die Gruppe ohne Einnahme von oralen Kontrazeptiva $(q-, n=39)$ und in die Oruppe mit Einnahme von oralen Kontrazeptiva $(q+, n=30)$. Zusammenstellung der in der Literatur beschriebenen Werte für die normale Ausscheidung der frelen Aminosăuren im 24-h-Urin von Erwachsenen; Angaben in $\mu \mathrm{mol} / \mathrm{l}, 0=$ Mittelwert \pm 1 Standardabweichung, p-Werte berechnet nach STUDENT

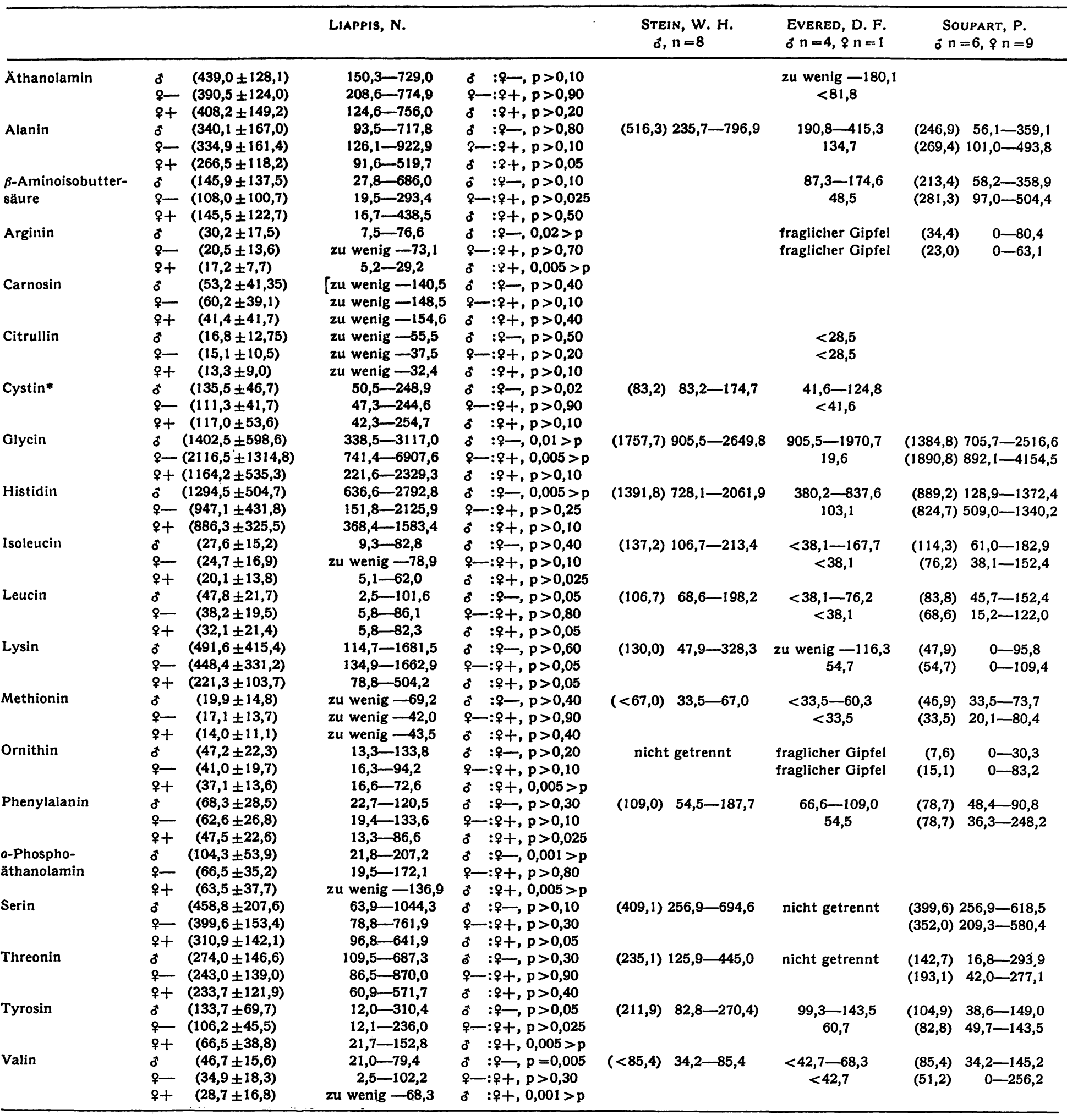

* Als $1 / 2$ Cystin berechnet

7. Mit Ausnahme von $\beta$-Aminoisobuttersäure, Cystin, Histidin, o-Phosphoäthanolamin und Threonin war die Ausscheidung der übrigen 15 untersuchten freien Aminosäuren im 24-h-Urin in der Gruppe der weiblichen Normalpersonen, die orale Kontrazeptiva ein- nahmen, niedriger als in der Gruppe der Frauen ohne Einnahme von Hormonen.

8. Signifikant niedriger im Vergleich zu der Gruppe der weiblichen Normalpersonen ohne Hormoneinnahme ergab sich in der Gruppe der weiblichen Normalper- 
Tab. 2

Ausscheidung der freien Aminosäuren pro Kilogramm Körpergewicht im 24-h-Urin gesunder Männer ( $n=33$ ) und Frauen ( $n=69)-$ aufgeteilt in die Gruppe ohne Einnahme von oralen Kontrazeptiva $(q-, n=39)$ und in die Gruppe mit Einnahme von oralen Kontrazeptiva $(q+, n=30)$. Zusammenstellung der in der Literatur beschriebenen Werte (29) $(n=8)$. Angaben in $\mu \mathrm{mol} / \mathrm{kg} / 24 \mathrm{~h},()=\mathrm{Extrembereich}$, p-Werte berechnet nach STUDENT

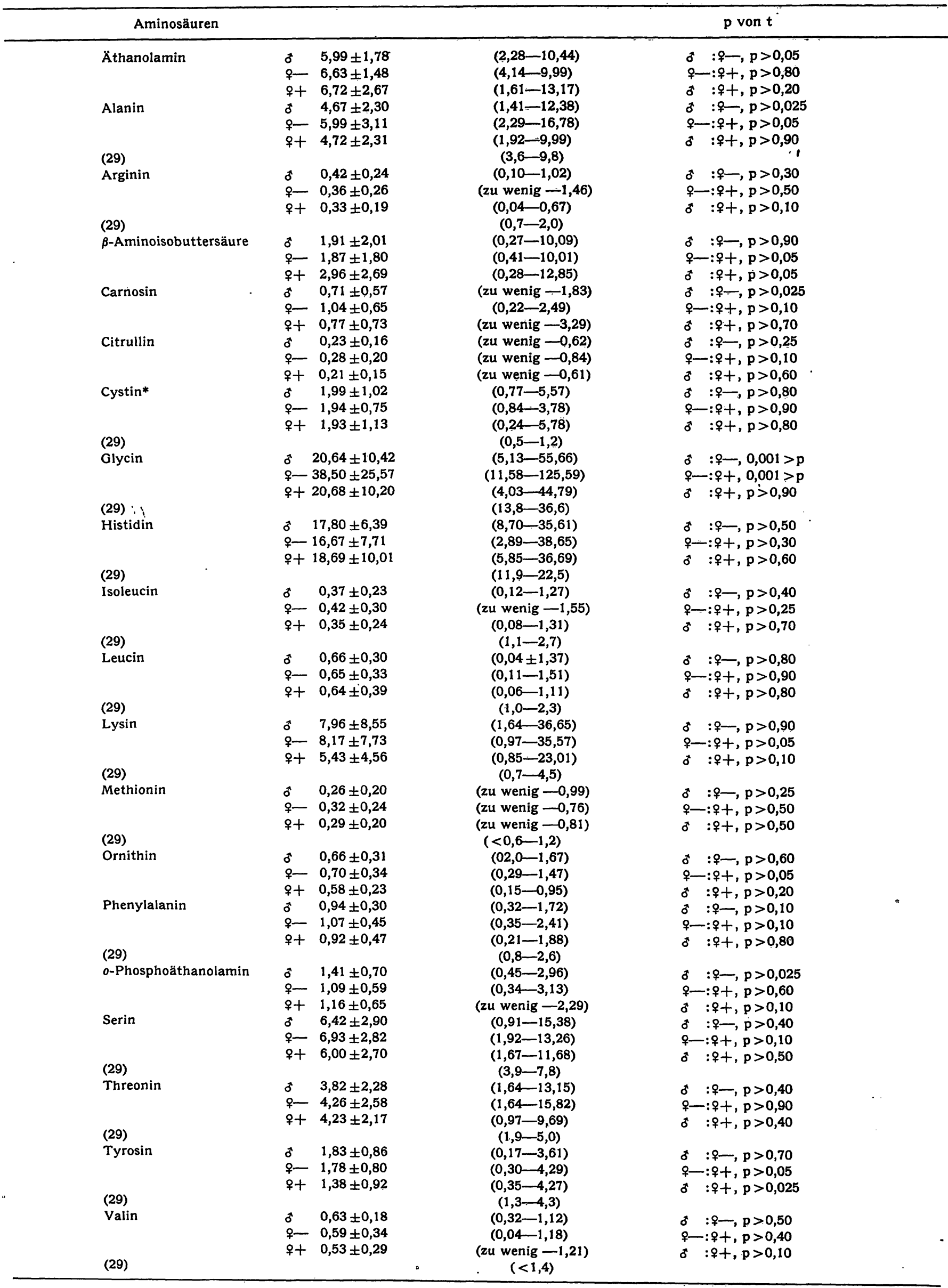

\footnotetext{
* Als 1/2 Cystin berechnet
} 
sonen mit Hormoneinnahme nur die Ausscheidung von Glycin.

9. Erheblich niedriger als bei den weiblichen Normalpersonen ohne Hormoneinnahme zeigte sich die Ausscheidung im 24-h-Urin von Alanin, Carnosin, Isoleucin, Lysin, Ornithin, Phenylalanin und Tyrosin bei diesen weiblichen Normalpersonen mit Hormoneinnahme.

10. Im Vergleich zu den männlichen (signifikant) und den weiblichen Normalpersonen ohne Hormoneinnahme war die Gesamtaminosäurenausscheidung bei den weiblichen Normalpersonen mit Hormoneinnahme seht stark erniedrigt.

11. In Übereinstimmung mit früheren Untersuchungsergebnissen ergab sich der Mittelwert des Körpergewichtes der männlichen Normalpersonen im Vergleich mit dem Mittelwert des Körpergewichtes der weiblichen Normalpersonen mit und ohne Hormoneinnahme signifikant erhöht.

12. Wurde die Ausscheidung der freien Aminosäuren im 24-h-Urin pro Kilogramm Körpergewicht berechnet, so ergab sich nur die Glycin-Ausscheidung der Frauen ohne Einnahme von oralen Kontrazeptiva im Vergleich zu den Männern und den Frauen mit Einnahme von oralen Kontrazeptiva signifikant vermehrt.

\section{Diskussion}

Quantitative Bestimmungen der Ausscheidung der freien Aminosäuren im 24-h-Urin von gesunden Erwachsenen wurden bisher mit Hilfe der Säulenchromatographie nur in geringer Anzahl durchgeführt.

Die in der Literatur beschriebenen Ergebnisse (23-25) sind in Tabelle 1 angeführt. STEIN (24) untersuchte 8 männliche Normalpersonen, EvEREd (25) 4 männliche und 1 weibliche Normalperson und Soupart (23) 6 männliche und 9 weibliche Normalpersonen.

Eine Zusammenfassung der in der Literatur beschriebenen Werte, die die Ausscheidung der freien Aminosäuren im 24-h-Urin gesunder Erwachsener $(n=8)$ pro Kilogramm Körpergewicht angeben (29), ist in Tabelle 2 dargestellt. Mit der beschriebenen Methode konnte Asparaginsäure und Glutaminsäure nicht exakt bestimmt werden, da Asparagin und Glutamin während der Enteiweißung des Urins und der säulenchromatographischen Analyse in nicht reproduzierbarem $\mathrm{Maße}$ hydrolysiert werden.

Wie in der vorliegenden Arbeit gezeigt werden konnte, ist die durchschnittliche Ausscheidưng bei den meisten der 20 untersuchten freien Aminosäuren im 24-h-Urin weiblicher Normalpersonen, die keine Hormonpräparate einnahmen, niedriger als bei den männlichen Normalpersonen. Es handelt sich um folgende 18 Aminosäuren: Äthanolamin, Alanin, $\beta$-Aminoisobuttersäure, Arginin, Citrullin, Cystin, Histidin, Isoleucin, Leucin, Lysin, Methionin, Ornithin, Phenylalanin, o-Phosphoäthanolamin, Serin, Threonin, Tyrosin und Valin.
Statistisch signifikant erniedrigt sind darunter Arginin, Histidin, o-Phosphoäthanolamin und Valin.

Unter dem Einfluß der oralen Kontrazeptiva verstärkt sich diese Tendenz insofern, als die Ausscheidung sämtlicher untersuchter freier Aminosäuren mit Ausnahme nur von $\beta$-Aminoisobuttersäure bei diesen Frauen niedriger ist als bei den Männern. Statistisch signifikant niedrigere Mittelwerte ergaben sich bei folgenden Aminosäuren: Arginin, Ornithin, o-Phosphoäthanolamin, Tyrosin und Valin.

Im Vergleich zu der männlichen Gruppe von Normalpersonen wurde von den Frauen ohne Hormoneinnahme nur Carnosin geringfügig und Glycin statistisch signifikant erhöht ausgeschieden. Dieser Befund korrelierte mit den Ergebnissen von Soupart (23).

Die Tendenz der niedrigeren Aminosäurenausscheidung, bedingt durch einen hormonellen Einfluß, ließ sich auch beim Vergleich der beiden Gruppen der Frauen nachweisen. Mit Ausnahme der geringfügig erhöhten Ausscheidung von $\beta$-Aminoisobuttersäure, Cystin, Histidin, o-Phosphoäthanolamin und Threonin wurden alle übrigen freien Aminosäuren von den Frauen, die orale Kontrazeptiva einnahmen, erniedrigt ausgeschieden. Statistisch sichern ließ sich dieses Ergebnis nur beim Glycin.

Gleiches Verhalten ergab sich ebenfalls bei der Untersuchung der Gesamtaminosäurenausscheidung im 24-hUrin von Erwachsenen und in der ausgeschiedenen Urinmenge dieser Normalpersonen. Die Urinausscheidung betrug bei den Männern (Durchschnittsalter: 31 Jahre und 11 Monate) 1355,8 \pm 442,9 ml - Extrembereich: 690 bis $2690 \mathrm{ml}$-, bei den Frauen ohne Hormoneinnahme (Durchschnittsalter: 31 Jahre und 10 Monate) $1127,4 \pm 422,6 \mathrm{ml}$ - Extrembereich: 490 bis $2260 \mathrm{ml}$ - und bei den Frauen mit Hormoneinnahme (Durchschnittsalter: 26 Jahre und 3 Monate) $980,0 \pm 449,1 \mathrm{ml}$ - Extrembereich: 320 bis $2290 \mathrm{ml}$-. Dieser Befund ließ sich zwischen der Gruppe der Männer und der Gruppe der Frauen mit Hormoneinnahme statistisch sichern.

Bei der Untersuchung der Gesamtaminosäurenausscheidung im 24-h-Urin war die Ausscheidung von 5675,2 士 $2266,4 \mu \mathrm{mol}$ bei den Frauen ohne Hormoneinnahme geringer als die Ausscheidung von 5858,8 $\pm 2224,0 \mu \mathrm{mol}$ bei den Männern. Dieser Unterschied ließ sich statistisch nicht sichern. Hingegen zeigte sich die Gesamtaminosäurenausscheidung im 24-h-Urin von $4537,3 \pm$ $2147,0 \mu \mathrm{mol}$ bei den Frauen mit Hormoneinnahme im Vergleich mit der Gesamtaminosäurenausscheidung der Männer statistisch signifikant niedriger. Zum Teil signifikante Abweichungen fanden ZinNeman et al. (30) auch bei der Untersuchung der freien Aminosäuren im Urin von 5 Männern nach Gabe von Diäthylstilböstrol. Signifikant erhöht zeigte sich die 1-Methylhistidin-Ausscheidung und signifikant erniedrigt die Tyrosin- und Histidin-Ausscheidung.

Im Vergleich zu dem Mittelwert des Körpergewichtes der männlichen Normalpersonen, ergab sich der Mittel- 
wert des Körpergewichtes der weiblichen Normalpersonen mit und ohne Hormoneinnahme signifikant erniedrigt.

Bei der Beurteilung der Aminosäurenausscheidung im 24-h-Urin ist das Bezugssystem von erheblicher Bedeutung. Die in der Literatur beschriebenen Werte wurden auf die Gesamtstickstoffausscheidung, Kreatininausscheidung, auf das Körpergewicht, die Körperoberfläche und auf den Anteil einer Aminosäure an der Gesamtaminosäurenausscheidung bezogen. Den physiologischen Erfordernissen würden am besten die Normalwerte entsprechen, die eigens für jedes Alter und für jedes Körpergewicht aufgestellt werden $(2,4$, 11, 23-25).

In der vorliegenden Arbeit wurde zusätzlich zu der geschlechtsspezifischen Untersuchung der Aminosäurenausscheidung pro $24 \mathrm{~h}$ noch das Körpergewicht der Versuchspersonen als Bezugssystem herangezogen. Anhand dieses Bezugssystems konnte folgender interessanter Befund erhoben werden. Bei der Berechnung der Ausscheidung der freien Aminosäuren im 24-hUrin pro Kilogramm Körpergewicht ergab sich, daß nur Glycin bei den Frauen ohne Einnahme oraler Kontrazeptiva in' Vergleich zu den Männern und den Frauen mit Hormoneinnahme statistisch signifikant erhöht ist. Bemerkenswert ist, daß alle übrigen statistisch signifikanten Unterschiede der beiden $\mathrm{Ge}$ schlechter, die nach Auswertung der 24-h-Ausscheidungswerte der freien Aminosäuren auftraten, sich nicht mehr bestätigten, wenn man die Ausscheidung pro Kilogramm Körpergewicht berechnete.

Das Körpergewicht und die Körperoberfläche, die als Bezugssystem in der Nephrologie verwendet wird und ebenso das Körpergewicht beinhaltet, zeigten sich also für den Vergleich der Aminosäurenausscheidung im Urin als besonders geeignet. Demzufolge ist es empfehlenswert, die Normalwerte der Aminosäurenausscheidung ${ }^{\top} \mathrm{im}$ ? 24-h-Urin in $\mu \mathrm{mol} / \mathrm{kg}$ Körpergewicht anzugeben. $\mathrm{Im}^{7}$ Gegensatz zu der bisher wenig untersuchten Aminosäurenausscheidung im Urin, besonders im Zusammenhang mit der Einnahme von oralen Kontrazeptiva, wurde der Einfluß der oralen Kontrazeptiva auf die Konzentration der freien Aminosäuren im Blutserum schon mehrfach untersucht. LANDAU et al. $(31,32)$ fanden nach intramuskulärer Gabe von Progesteron eine deutliche Abnahme des gesamten Aminosäurengehalts und der freien Aminosäuren im Plasma. Wie schon von Craft und Wrse $(33,34)$ beschrieben wurde, konnten wir (22) auch bei Frauen, die orale Kontrazeptiva einnahmen, niedrigere Aminosäuren-Konzentrationen im Blutserum feststellen. Bekannt ist auch das Auftreten von Veränderungen im Proteingehalt des Plasmas bei Frauen nach Einnahme von Östrogenen kombiniert mit Gestagenen (35) und von Östrogenen allein (36). Nicht unerwähnt sollte noch der Ernährungszustand, das Alter der Probanden (37) und der Nahrungseinfluß (38-41) für den Aminosäurenhaushalt des Menschen bleiben.

Eindeutig auf einen hormonell gesteuerten Mechanismus weist die generalisierte Hyperaminoacidurie, die während der Schwangerschaft beobachtet wird (42-45).

Die vorliegenden Ergebnisse der Ausscheidung der freien Aminosäuren im 24-h-Urin der untersuchten gesunden Männer und Frauen - berechnet pro Kilogramm Körpergewicht - sind unabhängig von dem beschriebenen hormonellen Einfluß (46), der sich bei der geschlechtsspezifischen Beurteilung der 24-h-Aminosäurenausscheidung zeigt. Mit Ausnahme von Glycin ergibt sich, bei Berücksichtigung des Körpergewichtes, ein einheitliches Aminosäurenspektrum im 24-h-Urin der Männer und Frauen.

Fräulein A. Jäkel, Ftau R. Igelbrink, Frau H. Bewerungè urd Fräulein C. KERN danke ich für die unermüdliche und gewissenhafte Mitarbeit. Ferner gilt mein Dank den Mitarbeiterr der Universitäts-Kinderklinik und der Medizinischen Universitätsklinik Bonn für die 24-h-Urinproben.

Die vorliegenden Untersuchungen wurden mit Unterstützung der Deutschen Forschungsgemeinschaft (Bonn-Bad Godesberg) durchgeführt.

\section{Literatur}

1. Bickel, H. \& Souchon, F. (1955), Arch. Kinderheilk., Beiheft 31, 1-151, Enke-Verlag, Stuttgart. - 2. Bigwood, E. J., CRO-' kaert, R., Schram, E., Soupart, P. \& Vis, H. (1959), Advances Clin. Chem. 2, 201. - 3. Chrsolm, J. J. (1959), J. Pediatrics 55, 303. - 4. Harris, H. (1955), Proc. 3rd Int. Congr. Biochem. Brüssel. - 5. Harrison, H. E. \& Harrison, H. C. (1954), Science 120, 606. - 6. Jonxis, J. H. P. (1957), Erg. Inn. Med. Kinderheilk. N. F. 8, 169. - 7. LINNEwEH, F. (1962), in Erbliche Stoffwechselkrankheiten, Verlag Urban und Schwarzenberg, München. - 8. SchreIER, K. (1963), in: Die angebotenen Stoffwechselanomalien, Verlag Georg Thieme, Stuttgart. - 9. SNYDerman, S. E. (1958), Pediatrics 21, 117. - 10. SNYDERMan, S. E. \& Horr, L. E. (1960), Advanc.' Pediat. 11,\$209. - 11. Soupart, P. (1960), Clin. Chim. Acta 5, 235. - 12. Nuederwieser, A. \& Curtius, H.-Ch. (1969), diese Z. 7, 404. - 13. Benson, J. W. (1970), in: New techniques in amino acid, peptide and protein analysis, Ann. Arbor Science Publ. Inc., Ann. Arbor, Michigan. - 14. Hamilton, P. B. (1966), in: Advances of chromatography, Vol. 2, 3-62, Verlag Marcel Dekker, New York.
15. Hamilton, P. B. (1968), in: Handbook of biochemistry, selected data for molecular biology, B-43 bis B-55, Verlag The chemical rubber Co., Cleveland, Ohio. - 16. Soupart, P. (1962), in: Amino acid pools, distribution, formation and function of free amino acids, 220-262, Elseviex Publ. Co., Amsterdam, London, New York. - 17. SteIN, W. H. \& MOORE, S. (1954), J. Biol. Chem. 211, 915. - 18. KING jr., J. S. (1964), Clin. Chim. Acta 9, 441. - 19. Breuer, J., Kaese, G. \& BreuER, H. (1968), diese Z. 6, 298. - 20. GeroK, W. (1960), Klin. Wochenschr. 38, 1212. - 21. OepeN, H. \& OEPEN, I. (1963), Klin. Wochenschr. 41, 1048. - 22. Liappis, N. (1972), diese Z. 10, 132. - 23. SouparT, P. (1959), Clin. Chim. Acta 4, 265. - 24. Strin, W. H. (1953), J. Biol. Chem. 201, 45. - 25. Evered, D. F. (1956), Biochem. J. 62, 416. - 26. Hamilton, P. B. (1963), Anal. Chem. 35, 2055. 27. PIEZ, K. A. \& Morris, L. (1960), Anal. Biochem. I, 187. 28. BroDEHL, J., JÄKEL, A. \& HAGGE, W. (1965), in: Automation in der analytischen Chemie, Technicon-Symposium, S. 473. 29. Documenta GeIGX (1969), in: Wissenschaftliche Tabellen, 7. Auf., S. 663, J. R. Geigy A. G., Verlag Pharma, Basel. - 
30. Zinneman, H. H., Musa, B. U. \& DoB, R. P. (1965), Metab. Clin. Exp. 14, 1214. - 31. LANDAU, R. L. \& LUGrBiHL, K. (1960), Recent Progr. Horm. Res. 17, 249. - 32. Landau, R. L. \& LUgIBrfu, K. (1967), Metab. Clin. Exp. 12, 1114. - 33. Craft, I. L. \& WISE, I. (1969), Lancet II, 1138. - 34. CRAFT, I. L. \& WISE, I. (1969), Nature (London) 222, 487. - 35. Laurell, C. B., KULLANDER, S. \& Thorell, J. (1968), Scand. J. Clin. Lab. Invest. 21, 337. - 36. Musa, B. U., Doz, R. P. \& Sear, U. S. (1967), J. Clin. Endocrinol. Metab. 27, 1463. - 37. Berger, H. (1956), Schweiz. Med. Wochenschr. 24, 711. - 38. McMenamy, R. H., Lund, C. C., Neville, G. J. \& Wallach, D. F. H. (1960), J. Clin. Invest. 39, 1675. - 39. SChreier, K. \& PlüCKthun, H.
(1950), Biochem. Z. 320, 447. - 40. Schreier, K. \& Plückthun, H. (1950), Z. Kinderheilk. 67, 480. - 41. Harding, R. S., SauberLICH, H. E. \& CANHAM, J. E. (1965), in: Automation in analytical chemistry, Technicon symposium, Sept. 8, New York, N. Y. 42. Soupart, P. (1959), Ann. Soc. Roy. Sci. Med. Nat. (Bruxelles) 12, 33. - 43. Bigwood, E. J., Schram, E., Soupart, P. \& Vis, H. (1961), Exposés Ann. Biochim. Méd. 23, 9. - 44. Hyrten, F. E. \& Cheyne, G. A. (1972), J. Obstet. Gynec. Brit. Commonwealth 79, 424. - 45. Zinneman, H. H., Seal, U. S. \& Doe, R. P. (1967), J. Clin. Endocrinol. Metab. 27, 397. - 46. LeCocQ, F. R., BRAdLEX, E. M. \& Goldzirher, J. W. (1967), Amer. J. Obstet. Gyncc. 99, 374.
Dr. rer. nat. N. Liappis Universitäts-Kinderklinik D-5300 Bonn

Adenauerallee 119 